\title{
Diagnosis of pulmonary aspergillosis using optical brighteners
}

\author{
S. Andreas*, S. Heindl*, C. Wattky*, K. Möller*, R. Rüchel**
}

\begin{abstract}
Diagnosis of pulmonary aspergillosis using optical brighteners. S. Andreas, S. Heindl, C. Wattky, K. Möller, R. Rüchel. (C) ERS Journals Ltd 2000.

ABSTRACT: Invasive pulmonary aspergillosis (IPA) is increasingly recognized especially in immunocompromised patients, but early diagnosis remains a problem. Fungal elements in clinical specimens can be directly stained with an optical brightener. The high intensity of the elicited fluorescence allows for rapid and reliable microscopic screening.

In the present study the authors aimed to validate this method. All specimens from bronchial secretions or pleural fluid suspected of mycosis $(n=94)$ and all bronchoalveolar lavages $(n=439)$ were prospectively evaluated by culture and staining with the optical brightener Blankophor-P-Flüssig.

IPA was diagnosed for 17 specimens from 13 patients, using a combination of clinical, culture and radiological data, and by biopsy $(n=3)$ or autopsy $(n=3)$. The overall incidence of IPA was $3.3 \%$. Nine of the 13 patients with IPA died (mortality $=69 \%$ ). Staining with the optical brightener and consecutive microscopic screening took $9 \pm 3$ $\mathrm{min}$. For the diagnosis of invasive aspergillosis, sensitivity was $\mathbf{0 . 8 8}$ and specificity was 0.99. Using culture, sensitivity was 0.76 and specificity was 0.99 .

In conclusion, direct examination of clinical specimens using the optical brightener has a high diagnostic potential for the diagnosis of invasive pulmonary aspergillosis. The reliability, simplicity and speed of the method render it suitable for routine diagnostic work.

Eur Respir J 2000; 15: 407-411.
\end{abstract}

\begin{abstract}
Depts of $*$ Cardiology and Pneumology, and **Microbiology, Georg-August-University, Göttingen, Germany.
\end{abstract}

Correspondence: S. Andreas

Dept of Cardiology and Pneumology

Robert-Koch-Str. 40

37075 Göttingen

Germany

Fax: 49551398918

Keywords: Aspergillus

blankophor

bronchoalveolar lavage

microscopical diagnosis

Received: May 201999

Accepted after revision October 71999
Invasive pulmonary mycoses are increasingly recognized especially in immunocompromised patients [1-3]. Early diagnosis of invasive pulmonary aspergillosis (IPA) improves prognosis, but remains a problem. Patients often have bleeding disorders which preclude invasive approaches. Culture from bronchial secretions or bronchoalveolar lavage (BAL) yields results only after some days. Furthermore, there is a high rate of false positives in the diagnosis of candidiasis $[4,5]$ and of false negatives in the diagnosis of IPA [1, 6-9]. Microscopic screening of the wet mount or after conventional staining (i.e. Gram, Giemsa) is time consuming and may be difficult because of low contrast $[4,10]$. Detection of fungal elements by polymerase chain reaction (PCR) from BAL specimens appears promising but is not readily available in the majority of diagnostic laboratories. Analogous considerations apply to Aspergillus antigen detection in blood or urine [11]. Generally the methods of nonculture diagnosis although encouraging, may be described as still investigational $[1,10,12,13]$. Improved management of IPA in neutropenic patients using early thoracic-computed tomography has been reported [9, 14]. Although the "halo sign" is suggestive of IPA, nonspecific findings are frequently found [15] and computer tomography raises logistic problems.

Fungal elements in clinical specimens can be directly stained with an optical brightener as described previously $[4,16-20]$. The high intensity of the elicited fluorescence allows for rapid microscopic screening at low magnifica- tions. However, this simple method is not routinely used in most laboratories and has not been formally evaluated. Therefore the current authors prospectively evaluated all specimens suspected of mycosis and all BAL by culture and staining with the optical brightener (Blankophor-Pflüssig; Bayer AG, Leverkusen, Germany).

\section{Methods}

\section{Protocol}

Between October 1994 and September 1996, all BAL specimens sent for microbiological evaluation $(n=439)$ from the authors' 1,500 bed university hospital were prospectively evaluated by culture and staining with the optical brightener. Furthermore all specimens suspected of mycosis $(n=94)$, as indicated by the physician taking care of the patient, were included in the study. Overall, the indication for the procedure was pulmonary infiltrates in immunocompromised hosts $(n=218)$ or in intubated patients $(n=$ 123 ). For the remaining patients the indication for investigation was the presence of pulmonary infiltrates unresponsive to conventional antibacterial antibiotic treatment. When clinically indicated physicians may have managed immunocompromised patients with pulmonary infiltrates without performing BAL or bronchial washings [21, 22]. The specimens were investigated immediately by direct staining with the optical brightener and consecutive 
microscopic screening by one of the authors (R. Rüchel) who was naive to the history and the results of other diagnostic procedures. The time needed for staining and thoroughly screening the specimens was recorded as were the result (graded in positive, negative, and the presumed pathogen). Thereafter, the physician submitting the specimen was informed of the results. Blood cultures, serology, chest radiograph and computed tomography of the thorax were obtained at the discretion of each patient's physician.

\section{Sample collection}

BAL was performed by fibreoptic bronchoscopy with slow infusion and aspiration of four $40 \mathrm{~mL}$ aliquots of sterile $0.9 \%$ saline solution with the bronchoscope wedged in a segmental bronchus at the site of maximal infiltrate on the chest radiograph [23]. Where diffuse infiltration was present the lingula or the right middle lobe was utilized. In patients with respiratory impairment BAL was carried out with lower fluid volumes as preferred by the operator. Bronchial washings were obtained in a number of intubated patients under sterile conditions after installing 10 $\mathrm{mL} 0.9 \%$ saline solution through a catheter.

\section{Staining}

Blankophor-P-flüssig (Bayer, Leverkusen, Germany) is an optical brightener of the diaminostilbene type, which has already been proposed for diagnostic use. Like other members of the group, it binds to $\beta$-glycosidically linked polysaccharides such as chitin or glucan [24]. The substance binds to similar targets as calcofluor white, but has a reduced tendency to precipitate in solution [25]. The aqueous stock solution of Blankophor is slightly alkaline $(\mathrm{pH} 10)$ since the compound is produced as a sodium salt. It is applied to clinical specimens after a 1,000-fold dilution in $20 \%$ potassium hydroxide solution (working solution). Smears were stained with one drop of the working solution on the mount directly before fluorescence microscopy as previously described [4]. The staining process proceeds rapidly on the complete mount. No fixation of the specimens on the mount, nor rinsing is required. Wet mounts yield best results, for once being dried on the slide, artefactual staining may occur. Fluorescence microscopy was performed with a Zeiss standard microscope equipped for epifluorescence, using a filter combination with excitation of $365-395 \mathrm{~nm}$ and a barrier of $240 \mathrm{~nm}$. Microphotographs were taken on conventional $35 \mathrm{~mm}$ film. Figure 1 shows a typical positive result.

\section{Further evaluation}

Cultures were performed by the standard technique [26]. Ten millilitres were centrifuged and portions of the pellet were inoculated on Sabouraud Dextrose agar and chocolate (heated-blood) agar with gentamicin and chloramphenicol. All cultures were incubated for 1 week and inspected daily. Aspergillus fumigatus antigen (galactomannan) was detected by the Pastorex-Aspergillus latex agglutination test using a monoclonal antibody (SanofiPasteur Diagnostica, Freiburg, Germany).

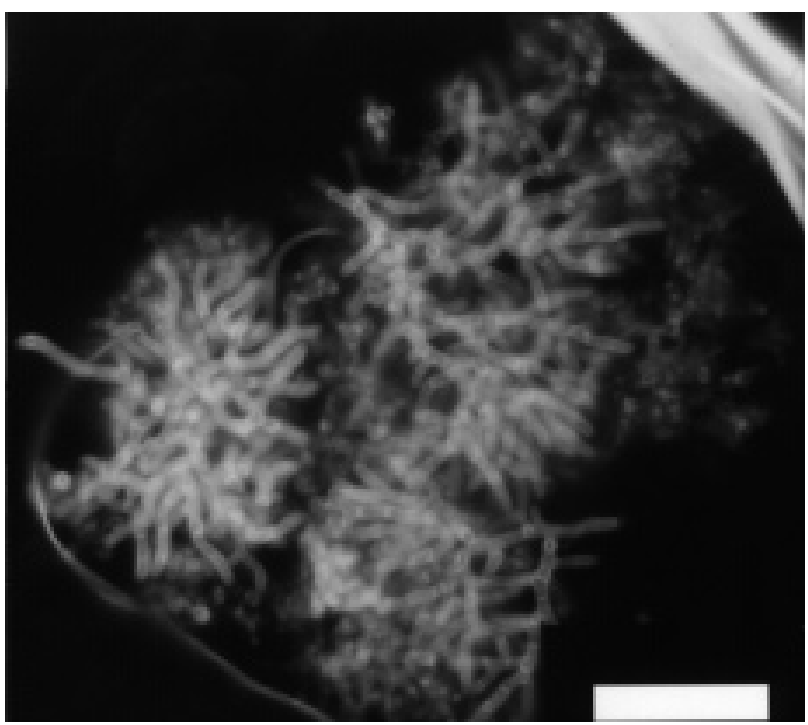

Fig. 1. - Aspergillus fumigatus stained with the optical brightener (Blankophor-P-flüssig) obtained by bronchoalveolar lavage. Internal scale bar $=5 \mu \mathrm{m}$.

\section{Definitions}

For the purposes of the study IPA was defined as: 1) identification of invasive fungal infection by biopsy or autopsy; 2) fungemia in immunocompromised hosts with pulmonary infiltrates and fever resistant to antibiotic treatment; 3) pulmonary infiltrates with identification of fungi in corresponding clinical specimens in immunocompromised hosts with fever resistant to antibiotic treatment; and 4) evidence of extrapulmonary mycosis in a patient with pulmonary infiltrates in a clinical setting predisposing to invasive mycosis. IPA was excluded when A. fumigatus was encountered in culture of blood or clinical specimens but 1) patients survived, revealed no pulmonary infiltrates and were by no means immunocompromised; 2) patients survived, no antimycotic treatment was instituted and the patients showed no clinical or radiological signs of invasive mycosis; or 3 ) there was no evidence of IPA at autopsy.

\section{Statistical analysis}

Sensitivity was defined as true positive results (TP)/(TP+ false negative results (FN)), the specificity as true negative results $(\mathrm{TN}) /($ false positive results $(\mathrm{FP})+\mathrm{TN})$, the positive predictive value as $\mathrm{TP} /(\mathrm{TP}+\mathrm{FP})$, and the negative predictive value as $\mathrm{TN} /(\mathrm{TN}+\mathrm{FN})$. These calculations were performed for the number of specimens, since specimens were obtained in some patients at different times of the underlying and infectious disease. Differences in proportions in categories were measured with Chi-squared analysis. Continuous values were given as mean \pm SD. Statistical analyses were performed on a personal computer using StatView (Abacus Inc., Berkeley, CA, USA). All p-values are two-tailed and a p-value $<0.05$ was considered statistically significant.

\section{Results}

\section{Subject characteristics}

Five hundred and thirty-three specimens from 398 patients, of whom 140 died (35 autopsies and 10 organ 
Table 1. - Subject characteristics

\begin{tabular}{lc}
\hline Parameter & $\mathrm{n}$ \\
\hline Age & $55 \pm 18$ \\
Intubated & $123(154)$ \\
Neutropenia $<1 \times 10^{3} \cdot \mathrm{mL}^{-1}$ & $38(45)$ \\
Glucocorticoids & $33(41)$ \\
Antifungal therapy & $94(125)$ \\
Amphotericin B & $40(58)$ \\
Fluconazole & $35(40)$ \\
5-flucytosine & $24(28)$ \\
\hline
\end{tabular}

Values are presented as the number of patients, with the number of specimens in parentheses.

biopsies) were analysed (table 1) Forty-eight specimens were from 17 patients with liver transplants, 12 from six patients with renal transplants, 45 from 30 patients with acute and 24 (16 patients) with chronic leukaemia, six multiple myeloma (four patients), one common variable immune deficiency syndrome, 30 (22 patients) symptomatic human immunodeficiency virus infection, 17 (11 patients) chronic alcoholics, and 125 from 111 patients with solid organ malignancies. Forty-one specimens were from 33 patients who were immunocompromised due to glucocorticoids. Rectal temperature was $>38.6 \mathrm{C}$ in $82 \%$ of the patients and $\mathrm{C}$ reactive protein was $138 \pm 125$ $\mathrm{mg} \cdot \mathrm{L}^{-1}$ in the 127 patients where data were available.

\section{Sampling of specimens}

The specimens were collected by BAL $(n=439)$, bronchial washings $(n=66)$, sputum $(n=15)$, lung biopsies $(n=8)$ and pleural fluid $(n=4)$. For 86 specimens silver-Grocott staining for the diagnosis of Pneumocystis carinii pneumonia and for 33 specimens cultures and PCR for the diagnosis of Cytomegalovirus infection were requested in addition to the above detailed work up. BAL was performed with $139 \pm 80 \mathrm{~mL}$ and a yield of $68 \pm 36 \mathrm{~mL}$. There were no significant differences between upper, middle or lower lobe sampling with respect to volume return (data not shown). Two patients with borderline respiratory failure were electively intubated following BAL. No pneumothorax, haemoptysis or death due to bronchoscopy or BAL were documented.

\section{Diagnosis of mycosis}

IPA was diagnosed for 17 specimens from 13 patients, using a combination of diagnostic modalities: clinical features, culture, radiological results, biopsy (in three patients) or autopsy (in three patients). The relevant features of these patients are summarized in table 2 . The overall incidence of IPA was $3.2 \%$ when calculated for the number of specimens and $3.3 \%$ for the number of patients. Nine of the 13 patients with IPA died (mortality $=69 \%$ ).

\section{Culture of specimens}

In diagnosing IPA using cultures of the specimens, four FN specimens (in three patients) and five FP (in five patients) were found. Sensitivity and specificity are given in table 3 . Positive predictive value was 0.72 and negative predictive value was 0.99 . When compared to the results obtained by staining there was no significant difference in sensitivity, specificity or negative and positive predictive values $P$. carinii pneumonia was detected in six, Cytomegalovirus pneumonia in two, and pulmonary nocardiosis in one patient.

\section{Optical brightener}

Staining with the optical brightener and consecutive microscopic screening (fig. 1) took $9 \pm 3$ min. Diagnosing IPA using the optical brightener revealed two FN specimens (in two patients) and two FP (in two patients).

Table 2. - Individual data of the 13 patients (17 specimens) with the diagnosis of invasive pulmonary aspergillosis

\begin{tabular}{|c|c|c|c|c|c|c|c|c|}
\hline $\begin{array}{l}\text { Patient } \\
\text { No. }\end{array}$ & Specimen & Diagnosis & Age & Sample & Blanko & Culture & Outcome & $\begin{array}{l}\text { Biopsy/ } \\
\text { autopsy }\end{array}$ \\
\hline 1 & & AML & 52 & BW & A & A & died & no \\
\hline 2 & & renal transplant, immunosupression & 62 & BW & A & A & surv. & no \\
\hline \multirow[t]{2}{*}{3} & $\mathrm{a}$ & Suicidal Zincphosphide- intoxication & 54 & BAL & A & A & died & autopsy \\
\hline & $\mathrm{b}$ & specimen b 3 days after specimen a & & BAL & A & A & & autopsy \\
\hline 4 & & Ewing's sarcoma & 13 & BAL & $\mathrm{A}, \mathrm{C}$ & $\mathrm{C}$ & surv. & no \\
\hline 5 & & Diabetes mellitus severe hypoglycaemia with coma & 76 & BAL & A & A & died & no \\
\hline 6 & & aplastic anaemia & 60 & Sputum & $\mathrm{A}, \mathrm{C}$ & $\mathrm{C}$ & died & biopsy \\
\hline 7 & & HIV positive, multiple myeloma & 48 & BAL & A & A & died & no \\
\hline \multirow[t]{2}{*}{8} & $\mathrm{a}$ & ALL & 48 & Sputum & A & - & died & autopsy \\
\hline & $\mathrm{b}$ & specimen b 8 days after & & BAL & A & - & & autopsy \\
\hline 9 & & AML & 41 & BAL & A & - & surv. & $\begin{array}{l}\text { biopsy } \\
\text { (see text) }\end{array}$ \\
\hline 10 & & tuberculosis & 64 & BW & A & $\mathrm{C}$ & surv. & no \\
\hline 11 & & septic shock & 72 & BAL & - & $\mathrm{C}$ & died & autopsy \\
\hline 12 & & Pneumonia/ multi-organ failure & 86 & BAL & $\mathrm{C}$ & $\mathrm{A}, \mathrm{C}$ & died & no \\
\hline \multirow[t]{3}{*}{13} & a & tuberculosis & 63 & BAL & A & A & died & biopsy \\
\hline & $\mathrm{b}$ & specimen b 7 days after specimen a & & BAL & A & A & & biopsy \\
\hline & c & specimen c 11 days after specimen a & & BW & A & - & & biopsy \\
\hline
\end{tabular}

Abbreviations: A: Aspergillus; C: Candida; -: negative; AML: acute meloid leukaemia; ALL: acute lymphoblastic leukaemia; BAL: bronchoalveolar lavage; BW: bronchial washing; HIV: human immunodeficiency virus; surv.: survivied. 
Table 3. - Sensitivity and specificity using culture and staining with the optical brightener Blankophor-P-Flüssig, for the diagnosis of invasive pulmonary aspergillosis

\begin{tabular}{lccc}
\hline & Technique & Sensitivity & Specificity \\
\hline Invasive pulmonary & & & \\
aspergillosis & Culture & 0.76 & 0.99 \\
& Blankophor & 0.88 & 0.99 \\
\hline
\end{tabular}

Sensitivity and specificity are given in table 3 . Positive predictive value was 0.88 and negative predictive value was 0.99 . One FN patient was on amphotericin B before and at the time of BAL and the diagnosis (chronic disseminated aspergillosis) was confirmed 2 yrs after BAL by biopsy of the chest wall (specimen No. 9 in table 2 ). The other FN patient (bone marrow toxicity due to pain killer abuse, thrombocytopenia but no neutropenia, gastrointestinal bleeding with shock and multiorgan failure) died 18 days after BAL (specimen No. 11 in table 2). Post-mortem revealed IPA of the left upper lobe. Specimens stained with the optical brightener were not checked for $P$. carinii which stained less intensively than fungi and are much smaller.

\section{Blood culture and serology}

Of the 290 blood cultures, there were four TP specimens in four patients for aspergillosis. There were six FN (in four patients), 280 TN (in 192 patients) but no FP. Aspergillus antigen was evaluated in 190 cases. There were four TP, eight FN and 175 TN.

\section{Discussion}

In this study, staining with the optical brightener Blankophor-P-Flüssig permitted the microscopic diagnosis of IPA within minutes after specimen collection. Staining sensitivity was as good as with cultured specimens.

In the current samples the diagnostic yield from staining was comparable to previous studies using conventional staining or cultures. KAHN et al. [7] reported that the presence of Aspergillus hyphae in BAL samples stained by Giemsa's, Gram's and Toluidine-O had a 53\% sensitivity and $97 \%$ specificity for the diagnosis of IPA, and cultures were positive in only four of 17 cases. In reviewing 21 immunocompromised patients with a diagnosis of IPA investigated using BAL and cytology, sensitivity was found to be $64.0 \%$ and specificity $99.1 \%$, while culture sensitivity was $40.0 \%$ and specificity $90.3 \%$ [8]. Poorer results were reported from an outbreak of aspergillosis in a general intensive care unit [27], or from non-neutropenic patients $[28,29]$. In the present study only two FP were encountered, although $>500$ specimens were stained, indicating a high specificity, not only in a sample with a relatively high prevalence of IPA, but also in a large sample with a low prevalence. It should be noted however, that microscopy does not allow unequivocal confirmation of IPA because other fungi (Pseudallescheria boydii, Fusarium species) may have similar appearances. In the present series the authors did not encounter such fungi. However, by microscopical criteria only, it is prudent to describe all Aspergillus-like fungal elements as hyalohyphomycetes if no typical conidiophors can be detected.

In the present study previous communications infrequently reported positive blood cultures for patients with IPA [30]. The poor sensitivity of Aspergillus antigen testing in detecting galactomannan by latex agglutination, which was encountered in the present study, is well known $[1,11,12]$. It should be noted that the current study was not designed to evaluate serology. Testing was performed only infrequently, at the discretion of each patient's physician, and it is well known that the detection of antigen is improved by testing frequently obtained samples [12, 31] and/or using a recently introduced enzyme linked immunosorbent assay (ELISA), which is based on the same monoclonal detector antibody as the agglutination test [32].

Mortality for the patients with IPA was $69 \%$ in the current series and comparable to previously published series [28]. Nevertheless mortality increases with the degree of immunosuppression [28].

A limitation of the present study is that autopsy or biopsy was performed in only $\sim 50 \%$ of the patients. In a strict sense the diagnosis of IPA necessitates tissue evidence of angioinvasion. However, an autopsy requires the consent of a family member and at the authors' institution as well as other hospitals this can be gained only infrequently. As discussed above ante-mortem biopsy is seldom possible. However, due to the prospective nature of the present study this limitation applies to the assessment of all diagnostic procedures evaluated. Therefore, the notion regarding the diagnostic potential of the optical brightener in relation to culture seems valid.

In conclusion, direct examination of bronchial washings or lavage with the optical brightener Blankophor-P-Flüssig has diagnostic value in the diagnosis of invasive pulmonary aspergillosis. The reliability, simplicity and speed of the method render it suitable for diagnostic routine.

\section{References}

1. Denning DW. Invasive aspergillosis. Clin Infect Dis 1998; 26: 781-805.

2. Bodey G, Bueltmann B, Duguid W, et al. Fungal infections in cancer patients: an international autopsy survey. Eur J Clin Microbiol Infect Dis 1992; 11: 99-109.

3. Haron E, Vartivarian S, Anaissie E, Dekmezian R, Bodey GP. Primary Candida pneumonia. Experience at a large cancer center and review of the literature. Medicine Baltimore 1993; 72: 137-142.

4. Rüchel R, Margraf S. Rapid microscopical diagnosis of deep-seated mycoses following maceration of fresh specimens and staining with optical brighteners. Mycoses 1993; 36: 239-242.

5. Saito H, Anaissie EJ, Morice RC, Dekmezian R, Bodey GP. Bronchoalveolar lavage in the diagnosis of pulmonary infiltrates in patients with acute leukemia. Chest 1988; 94: 745-749.

6. Bretagne S, Costa JM, Marmorat Khuong A, et al. Detection of Aspergillus species DNA in bronchoalveolar lavage samples by competitive PCR. J Clin Microbiol 1995; 33: 1164-1168.

7. Kahn FW, Jones JM, England DM. The role of bronchoalveolar lavage in the diagnosis of invasive pulmonary aspergillosis. Am J Clin Pathol 1986; 86: 518-523. 
8. Levy H, Horak DA, Tegtmeier BR, Yokota SB, Forman SJ. The value of bronchoalveolar lavage and bronchial washings in the diagnosis of invasive pulmonary aspergillosis. Respir Med 1992; 86: 243-248.

9. von Eiff M, Zuhlsdorf M, Roos N, Hesse M, Schulten R, van de Loo J. Pulmonary fungal infections in patients with hematological malignancies-diagnostic approaches. Ann Hematol 1995; 70: 135-141.

10. Walsh TJ, Lyman CA, Pizzo PA. Laboratory diagnosis of invasive fungal infections in patients with neoplastic diseases. In: Meunier F, ed. Clinical Infectious Diseases. London, UK, Bailliere Tindall, 1995; pp. 25-70.

11. Rogers TR, Haynes KA, Barnes RA. Value of antigen detection in predicting invasive pulmonary aspergillosis. Lancet 1990; 336: 1210-1213.

12. de Repentigny L. Serodiagnosis of candidiasis, aspergillosis, and cryptococcosis. Clin Infect Dis 1992; 14 (Suppl. 1): S11-S22.

13. van Rens MTM, Vernooy-Jeras R, Merton-de Ridder M, van Velzen-Blad H, Van den Bosch JMM. Detection of immunoglobulins G and A to Aspergillus fumigatus by immunoblot analysis for monitoring Aspergillus induced lung disease. Eur Respir J 1998; 11: 1274-1280.

14. Caillot D, Casasnovas O, Bernard A, et al. Improved management of invasive pulmonary aspergillosis in neutropenic patients using early thoracic computed tomographic scan and surgery. J Clin Oncol 1997; 15: 139-147.

15. Logan PM, Primack SL, Miller RR, Muller NL. Invasive aspergillosis of the airways: radiographic, CT, and pathologic findings. Radiology 1994; 193: 383-388.

16. Hageage GJ, Harrington BJ. Use of calcofluor white in clinical mycology. Laborat Med 1984; 15: 109-112.

17. Hollander H, Keilig W, Bauer J, Rothemund E. A reliable fluorescent stain for fungi in tissue sections and clinical specimes. Mycopathologia 1984; 88: 131-134.

18. Monheit JE, Cowan DF, Moore DG. Rapid detection of fungi in tissues using calcofluor white and fluorescence microscopy. Arch Pathol Lab Med 1984; 108: 616-618.

19. Wachsmuth ED. A comparison of the highly selective fluorescence staining of fungi in tissue sections with Uvitex 2B and Calcofluor White M2R. Histochemical Journal 1988; 20: 215-221.

20. Aslanzadeh J, Stelmach PS. Detection of Pneumocystis carinii with direct fluorescence antibody and calcofluor white stain. Infection 1996; 24: 248-250.
21. Pizzo PA. Management of fever in patients with cancer and treatment-induced neutropenia. N Engl J Med 1993; 328: $1323-1332$.

22. Maschmeyer G, Link H, Hiddemann W, et al. Pulmonary infiltrations in febrile patients with neutropenia. Risk factors and outcome under empirical antimicrobial therapy in a randomized multicenter study. Cancer 1994; 73 : 2296-2304.

23. European Society of Pneumology Task Group. Technical recommendations and guidelines for bronchoalveolar lavage (BAL). Report of the European Society of Pneumology Task Group. Eur Respir J 1989; 2: 561585.

24. Wood PJ, Fulcher RG. A basis for specific detection and histochemistry of polysaccharides. J Histochem Cytochem 1983; 31: 823-826.

25. Monod M, Baudraz-Rosselet M, Ramelet AA, Frenk E. Direct mycological examination in dermatologie: a comparison of different methods. Dermatologica 1989; 179: 183-186.

26. Merz WG, Roberts GD. Detection and Recovery of Fungi from Clinical Specimens. In: Murray PR, ed. Manual of Clinical Microbiology. 6th Edn. Washington, D.C., ASM Press, 1995; 709-722.

27. Humphreys H, Johnson EM, Warnock DW, Willatts SM, Winter RJ, Speller DC. An outbreak of aspergillosis in a general ITU. J Hosp Infect 1991; 18: 167-177.

28. Yu VL, Muder RR, Poorsattar A. Significance of isolation of Aspergillus from the respiratory tract in diagnosis of invasive pulmonary aspergillosis. Results from a three-year prospective study. Am J Med 1986; 81: 249254.

29. Horvath JA, Dummer S. The use of respiratory-tract cultures in the diagnosis of invasive pulmonary aspergillosis. Am J Med 1996; 100: 171-178.

30. Sharma OP, Chwogule R. Many faces of pulmonary aspergillosis. Eur Respir J 1998; 12: 705-715.

31. Edwards JE Jr., Masur H, Rosen PP, Armstrong D. Pulmonary disease caused by Candida species. $N$ Engl $J$ Med 1991; 324: 1060-1062.

32. Herrmann J, Grugel A, Freidank H, Bertz H, Finke J. Aspergillus-Antigen-Nachweis: Vergleich eines neuen Sandwitch-ELISA mit dem Latex-Agglutinationstest bei Patienten mit histologisch gesicherter invasiver Aspergillose. Mycoses 1998; S1: 83-85. 